\title{
Single-Electrode Triboelectric Nanogenerator for Scavenging Friction Energy from Rolling Tires
}

\author{
Yanchao Mao, ${ }^{a, b}$ Dalong Geng, ${ }^{a}$ Erjun Liang, ${ }^{b}$ Xudong Wang ${ }^{\mathrm{a}^{*}}$ \\ ${ }^{\mathrm{a}}$ Department of Materials Science and Engineering, University of Wisconsin-Madison, Madison, \\ WI 53706, USA \\ ${ }^{\mathrm{b}}$ Key Laboratory of Materials Physics of Ministry of Education of China, School of Physical \\ Science \& Engineering, Zhengzhou University, Zhengzhou 450052, China \\ *Email: xudong@engr.wisc.edu
}

\begin{abstract}
Triboelectric nanogenerator (TENG) is a novel energy harvesting device to convert mechanical energy into electricity based on the universally known triboelectric principle. In this work, we demonstrated an innovative design of single-electrode TENG (S-TENG) using PDMS to simulate the tire surfaces for scavenging the wasted friction energy from rolling tires. By fixing the PDMS S-TENG on a rubber wheel, the performance of scavenging friction energy was systematically investigated. The electric output of the S-TENG-on-wheel monotonically increased with the increase of the moving speed and weight load of the wheel. The maximum instantaneous power was obtained to be $1.79 \mathrm{~mW}$ at a load resistance of $10 \mathrm{M} \Omega$, corresponding to the highest energy conversion efficiency $10.4 \%$. Multiple S-TENGs were implemented to the tires of a toy vehicle and instantaneously powered 6 commercial green light emitting diodes (LEDs) while the vehicle was moving on the ground. This successful demonstration provides a promising solution to scavenge the wasted friction energy from rolling tires, which may improve the fuel efficiency or the cruising ability of electric vehicles.
\end{abstract}

Keywords: Triboelectric nanogenerator; Mechanical energy harvesting; PDMS; Rolling tires; Wasted friction energy 


\section{Introduction}

Due to the rapid-growing fossil fuel consumptions and global energy demands in the past decades, intensive research efforts have been devoted to the development of alternative renewable energy technologies. Harvesting mechanical energy from ambient environment is considered as an effective and promising approach to supplement the energy needs due to its great abundance and ubiquity.[1-3] In 2006, nanogenerator (NG) was developed using piezoelectric nanomaterials or nano-composites to harvest mechanical energy from various mechanical energy sources such as fluid or air flows, $[4,5]$ ultrasonic waves,[6] deformations, rotations, [7, 8] and human activities.[9] Most recently, Based on the coupling of triboelectric friction and electric induction, triboelectric nanogenerator (TENG) has been developed as a new principle for scavenging mechanical energy with orders-of-magnitude-enhanced electric output. Due to its simple design and broad material selection, TENG has quickly shown great promises as a power source for a wide range of applications, such as powering portable electronics,[2] self-powered sensors,[10] charging lithium ion batteries,[11] electrodeposition,[12] electrodegradation of dyes,[13] and electrolyzing water.[14]

One of the most attractive merits of NG is its capability of scavenging the wasted mechanical energy in many different forms. Friction is a major energy loss in a rotation system, where rolling tires on the ground is the most common example. Statistics conducted by the US Department of Energy discovered that $5-7 \%$ of energy was consumed by the rolling resistance in vehicles (not including breaking loss).[15] Harvesting this type of energy would be promising in improving the fuel efficient or the mileage per charge of electric vehicles. TENG has a desire configuration for harvesting energy from friction.[16] However, most reported TENGs employ a design that consists of a pair of thin film materials with different electron affinity and is coated with metal electrodes to output the induced charges.[17] This design imposes limitations in harvesting the friction energy from a tire when it's continuous rolling on the ground. Abrasion from the ground would quickly damage the functioning part. This challenge would be solved if the ground surface could act as one charge-generating material and the tire surface acts as the other one. Most materials on the ground surface like silica, cement, and even metals are electron donating materials.[18] When they are in contact with electron accepting materials such as polymer and rubber, opposite charges can be induced on both surfaces, and thus the triboelectric principle would apply. Polydimethylsiloxane (PDMS) is a flexible polymer with strong electron 
attracting ability, which has been successfully used in a variety of TENG development.[19-21] In this paper, we report a novel single-electrode TENG (S-TENG) design using rough PDMS thin films to simulate the tire surfaces for scavenging the wasted friction energy from rolling tires. By fixing the PDMS S-TENG on a rubber wheel, the friction energy scavenging capability was demonstrated and systematically investigated. Such a S-TENG device was implemented to the tires of a toy vehicle and instantaneously powered 6 commercial light emitting diodes (LEDs) while the vehicle was moving on the ground. This development offers a promising solution to recovering the friction energy from rolling tires, which may find a practical application potential in improving the vehicle's fuel efficiency, particularly for electrical cars.

\section{Experimental Section}

Fabrication of PDMS S-TENG: A piece of sandpaper was taped at the bottom of a polystyrene plastic dish. Liquid PDMS elastomer and cross-linker were mixed with a ratio of 10:1 and then uniformly casted on the sandpaper. After $20 \mathrm{~min}$ degasing in vacuum and a thermal incubation at $90{ }^{\circ} \mathrm{C}$ for 1 hour, a uniform PDMS thin film with a rough surface was formed. The film was then peeled off from the sandpaper. A piece of $\mathrm{Cu}$ foil with one side conductive adhesive was attached to the flat side of the PDMS thin film acting as the electrode to conduct the induced charges. Figure S1 shows the SEM image of the sand paper mold surface. There are many micro peaks with sharp tips locate on the sand paper surface. These tips result in micro pores and rough surface of the PDMS thin film. The entire surface morphology corresponds to that of the prepared PDMS thin film.

S-TENG design and characterization: A LEO 1530 scanning electron microscope and Park XE-70 atomic force microscopy (AFM) were used to characterize the surface feature of the PDMS thin film. A S-TENG film with a size of $1 \times 1 \mathrm{~cm}^{2}$ was attatched onto the surface of a rubber wheel $(7 \mathrm{~cm}$ in diameter) with the rough surface facing outward. The back electrode was connected to the metal rim on the wheel for induced charge collection. The total weight of the wheel is $0.5 \mathrm{~kg}$. The wheel was driven by a computer-controlled linear actuator with controlled linear speeds. A polyoxymethylene plastic bar $(61 \mathrm{~cm}$ in length) was added between the actuator and wheel to avoid the electric-field noise generated from the electromagnetic actuator. The electrical outputs of the TENG were measured using an Autolab PGSTAT302N station and an Agilent DSO1012A oscilloscope. 
S-TENG on vehicle demonstration: 6 PDMS S-TENGs $\left(1.5 \times 3.5 \mathrm{~cm}^{2}\right.$ each $)$ were attached onto the tire surface of a toy car (each back wheel has 3 S-TENGs). A piece of Al foil was placed on the bottom of the toy car as the reference electrode. Commercial green LEDs connected in series were installed on the toy car as the vehicle's headlights. Another electrode of the LED was connected to the reference electrode, which is equivalent to ground connection.

\section{Results and Discussion}

The S-TENG design starts from the fabrication of a flexible PDMS thin film with rough friction surface. Liquid PDMS elastomer and cross-linker were mixed and uniformly casted on a simple piece of sandpaper mold. After thermal incubation, a flexible PDMS thin film with a rough surface was formed. Figure 1a shows a scanning electron microscopy (SEM) image of the PDMS thin film surface, revealing its uniform microstructured rough surface. The as-fabricated thin film is flexible and stretchable (inset of Figure 1a), which allows it to be seamlessly attached onto the tire surface. The topography of the PDMS surface was further characterized using atomic force microscopy (AFM). As illustrated in Figure 1b, the pore depths are about several micrometers, and the entire surface topography is consistent with that of the sand paper mold (Figure S1). The rough surface of the PDMS thin film can enlarge the effective contact surface area in friction, and thus is beneficial to the electrical output.[22] Then, a layer of metal electrode ( $\mathrm{Cu}$ foil in this case) was attached onto the flat backside of the PDMS thin film for charge collection.

The experimental design for measuring the energy harvesting ability of the S-TENG from a rolling tire is schematically shown in Figure 1c (see setup details in the Method Section). The flexible S-TENG $(1 \mathrm{~cm} \times 1 \mathrm{~cm} \times 120 \mu \mathrm{m})$ was attatched onto the surface of a rubber wheel $(7$ $\mathrm{cm}$ in diameter). A metal rim was placed on the wheel and connected to the S-TENG for collecting output signals. The wheel was driven by a computer-controlled linear actuator, which provided linear motions at different speeds. Rotating motions of the wheel make the S-TENG on its surface periodically contacting the supporting surface and then separate. Figures $1 \mathrm{~d}$ and $1 \mathrm{e}$ present the open-circuit voltage $\left(V_{\mathrm{OC}}\right)$ and short-circuit current density $\left(J_{\mathrm{SC}}\right)$ from the S-TENG when the wheel was moving at a linear speed of $0.3 \mathrm{~m} / \mathrm{s}$. The average peak values of the $V_{\mathrm{OC}}$ and $I_{\mathrm{SC}}$ reached $2.3 \mathrm{~V}$ and $2.0 \mu \mathrm{A}$, respectively. As shown in the insets of Figures $1 \mathrm{~d}$ and $1 \mathrm{e}$, the 
voltage and current output signals generated in one contact-separation cycle exhibited a similar pattern.

The electricity generation mechanism of the S-TENG on a rolling wheel is schematically shown in Figure 2a. Once the PDMS thin film is brought to contact with the ground, electrons are transferred from the ground into the surface of PDMS. The charge transfer is a direct result of the different electron affinity between PDMS and other materials that could be a ground (such as $\mathrm{SiO}_{2}$, metal, wood, etc.). This will leave the PDMS with negative charges and the ground surface with positive charges (step i). Upon further rotating, the PDMS thin film fully contacts the ground surface. At this moment, the charge transfer is completed and the opposite charges on the two contact surfaces are in electrical equilibrium (step ii). No current flows though the external load. As the charged PDMS surface continuously rotates away from the ground, the overlapped area between the charged PDMS and ground surface decreases and the charges on the PDMS can no longer be fully balanced by the ground. The unbalanced negative charges on the PDMS surface drive a current flow from the reference electrode (a piece of metal sheet) to the back electrode of the PDMS film producing a negative current pulse (step iii). The induced current maximizes when the PDMS film completely leaves the ground surface, where the induced positive charges equal to the amount of the negative charges on the PDMS surface (step iv). Subsequently, continue rotation of the wheel brings the PDMS film back to ground again contact the ground surface again (note: the contacting point is a new position on the ground), and all the charges remaining statistic charge will be released to the ground. Simultaneously, the induced positive charges on the back electrode become unbalanced and drive current flow back to the reference electrode producing a positive current pulse (step v). Then the system comes back to its original state and goes through these steps again producing continuous electric pulses as shown in Figure 1d and e.

Figure $2 \mathrm{~b}$ presents a typical electric output pattern through charging/discharging cycle. Each step along the current curve is marked accordingly. It should be noted that between steps iv and v, the wheel goes through one circle in air. This may course a certain amount of surface charge lose. As a result, the positive charges generated upon PDMS re-contacting the ground are less than the negative charges induced by separating PDMS from the ground. The corresponding number of induced charge was calculated by the integral area of the I-t curve in Figure $2 b$ through following equation: 


$$
q=\int_{a}^{b} I d t
$$

where $q$ is the induced charge, $I$ is the output current, $t$ is the time, and $[\mathrm{a}, \mathrm{b}]$ is the time interval. As shown in Figure 2c, from point $\mathrm{v}$ to iii, the charge increased from 0 to $4.6 \mathrm{nC}$ when the PDMS film re-contacts the ground, suggesting the releasing of electrons from the PDMS surface. After the electrical equilibrium state at point iii, the charge decreased to $-1.3 \mathrm{nC}$ at point ii in the separating process. In the entire electricity generation process cycle, the generated negative charges $(5.9 \mathrm{nC})$ are more than the positive charges $(4.6 \mathrm{nC})$. The difference represents the loss of charge during wheel rotation and is a typical feature for all output peaks (Figure S2).

Considering the most relevant functions of a wheel, the influences of moving speed and weight load of the wheel on the output performances were investigated. The average peak $V_{\mathrm{OC}}$ was plotted as a function of the linear moving speed of the wheel as shown in Figure 3a (voltage outputs are shown in Figure S3). The peak $V_{\mathrm{OC}}$ increased from $0.3 \mathrm{~V}$ to $4.2 \mathrm{~V}$ as the moving speed increased from $0.1 \mathrm{~m} / \mathrm{s}$ to $0.5 \mathrm{~m} / \mathrm{s}$. A clear linear relationship between the average peak $V_{\mathrm{OC}}$ and the speed was obtained yielding a slop of $9.7 \mathrm{~V} \mathrm{~m}^{-1} \mathrm{~s}$. The linear increasing of the electric output can be attributed to the raised contacting and separation rates of the friction surfaces that would induce more accumulative charges within the same time frame.[23, 24] To investigate the influence of weight load on the S-TENG's performance, a series of extra weights from 0.3 to 2.2 $\mathrm{kg}$ were added onto the wheel. Figure $3 \mathrm{~b}$ shows the average peak $V_{\mathrm{OC}}$ of the S-TENG as a function of load; while the wheel was moving at a constant linear speed of $0.3 \mathrm{~m} / \mathrm{s}$ (see voltage outputs in Figure S4). With the extra load increased from 0 to $2.2 \mathrm{~kg}$, the average peak $V_{\mathrm{OC}}$ linearly increased from $2.3 \mathrm{~V}$ to $3.7 \mathrm{~V}$. From the fitted curve, every $1 \mathrm{~kg}$ load would increase the average peak $V_{\mathrm{OC}}$ by $\sim 0.7 \mathrm{~V}$. The load enhancement can be attributed to the larger pushing-down force from heavier loads. In general, larger force yields larger contacting area between the STENG and ground surface. Microscopically, the surface of PDMS film has many microstructured features. These features can provide high surface area, but they may not become effective until they are squeezed down. Meanwhile, the ground surface is also rough and would leave many small gaps between these two rough surfaces. With a larger pushing-down force, the PDMS can deform and fill more gaps due to its good elastic property, leading to a larger contact area. Macroscopically, the contact area between a wheel and the ground is a curved surface. Larger force can flatten the contact surface between the rubber wheel and solid ground to a 
greater extent, and thus significantly enlarge the contact area for higher output. It is expected that the electrical output would reach a theoretical saturation point when all of the vacant space is completely filled by the intimate contact between the wheel and group.

Similar to other TENG devices, the electric output of the S-TENG on wheel is also a function of the load resistance, through which the output power can be optimized. Figure 3c shows the resistance-dependent output voltage and current when the load resistor was swept from $0.1 \mathrm{M} \Omega$ to $100 \mathrm{M} \Omega$ (the wheel was moving at a constant speed of $0.3 \mathrm{~m} / \mathrm{s}$ with none extra weight loading). The peak $V_{\mathrm{OC}}$ increased from a few volts to nearly 700 volts as the resistant increased to $100 \mathrm{M} \Omega$. Meanwhile, the peak $I_{\mathrm{SC}}$ followed an opposite trend and decreased from $\sim 8 \mu \mathrm{A}$ to $\sim 2$ $\mu \mathrm{A}$. The instantaneous output power was calculated from the products of the peak voltage and current, and plotted as a function of the resistance (Figure 3d). The maximum output power was found to be $1.79 \mathrm{~mW}$ at a load resistance of $10 \mathrm{M} \Omega$, corresponding to a power density of 14.92 $\mathrm{mW} / \mathrm{cm}^{3}$ for PDMS films. The energy conversion efficiency $(\eta)$ can be calculated from the following equation:

$$
\eta(\%)=U I / C_{\mathrm{RRC}} W v
$$

where $C_{\mathrm{RRC}}$ is rolling resistance coefficient (RRC, 0.007 for a typical tire[25]), $W$ is the weight ( $W=4.9 \mathrm{~N}$ at zero extra load), and $v$ is the speed. The highest efficiency reaches $10.4 \%$ at the load resistance of $10 \mathrm{M} \Omega$.

The output from the wheel rolling can be enhanced by adding multiple S-TENGs on the wheel surface. Figures 4a-d show the current outputs when 1, 2, 3, and 4 PDMS S-TENG units were attached on the same wheel, respectively. The back electrodes of all the S-TENG were electrically separated. The total counts of current output peaks in a single rotation cycle are consistent with the unit quantity. The number of PDMS units has negligible influence on the maximum $I_{\mathrm{SC}}$ peak value, which stayed at $\sim 2.7 \mu \mathrm{A}$. The total number of charge generated in a rotation cycle is calculated based on the current curve and plotted in Figure 4e. The total induced charge increased monotonically from $31.8 \mathrm{nC}$ to $60.6 \mathrm{nC}$ as the number of PDMS units increased from 1 to 4 . This charge enhancement is expected to saturate as the entire wheel surface is covered by S-TENG unit.

The successful development of PDMS S-TENG on wheels for friction energy harvesting suggests it can be an inherent part of a tire owing to the single electrode design. As a demonstration, 6 PDMS S-TENGs $\left(1.5 \times 3.5 \mathrm{~cm}^{2}\right.$ each $)$ were attached onto the tire surface of a 
toy car (each back wheel has 3 S-TENGs). The flexible feature of the S-TENG allows it to be well fitted with the tire profile (inset of Figure 4f). 6 commercial green LEDs connected in series were used as the vehicle's headlights (Figure 4f). A piece of Al foil was placed on the bottom of the vehicle as the reference electrode. When the vehicle was moving on the ground, the generated electricity through triboelectric friction could instantaneously light up 2 LED lights (see Video S1 in the Supporting Information). The equivalent circuit for powering LEDs is shown in Figure S5. If a certain amount of additional force was applied to the vehicle by hand pushing when it was moving, the 6 LED headlights could be powered simultaneously, as shown in Figure 4g and Video S2 (see the Supporting Information). It should also be noted that in a practical tire system, the tire surface itself can serve as the PDMS surface. Implanting back electrodes inside the tire body can realize the same device configuration and function as a PDMS S-TENG.

\section{Conclusion}

In summary, we demonstrated an innovative design of S-TENG based on the triboelectric principle using PDMS thin films with a rough surface. The PDMS only has one back electrode for induced charge collection and its front surface is in direct contact with the supporting ground. This single electrode design offers a unique capability for scavenging the otherwise-wasted friction energy between a rolling wheel and the ground. The electric output from the S-TENGon-wheel increased monotonically following the increase of the moving speed and weight load. By optimizing the load resistance on the external electric circuit, the maximum instantaneous power was found to be $1.79 \mathrm{~mW}$ at a load resistance of $10 \mathrm{M} \Omega$, corresponding to the highest energy conversion efficiency of $10.4 \%$. Multiple S-TENGs on the wheel surface can effectively enlarge the number of induced charges, and thus improve the electric output power. Operation of S-TENGs on the wheels of a toy car was also demonstrated. When the vehicle was moving on the ground, the S-TENG generated electricity could instantaneously power 6 commercial green LEDs without any energy storage component. The successful development of PDMS S-TENG demonstrated a promising application capabitliy for scavenging the wasted friction energy between the tires and ground from a running vehicle, and thus improves the fuel efficiency or the total mileage per charge of electric vehicles. 


\section{Acknowledgments}

The authors thank financial support from National Science Foundation under Award CMMI1148919 and Air Force under Award FA9550-13-1-0168.

Figures and Figure Captions:
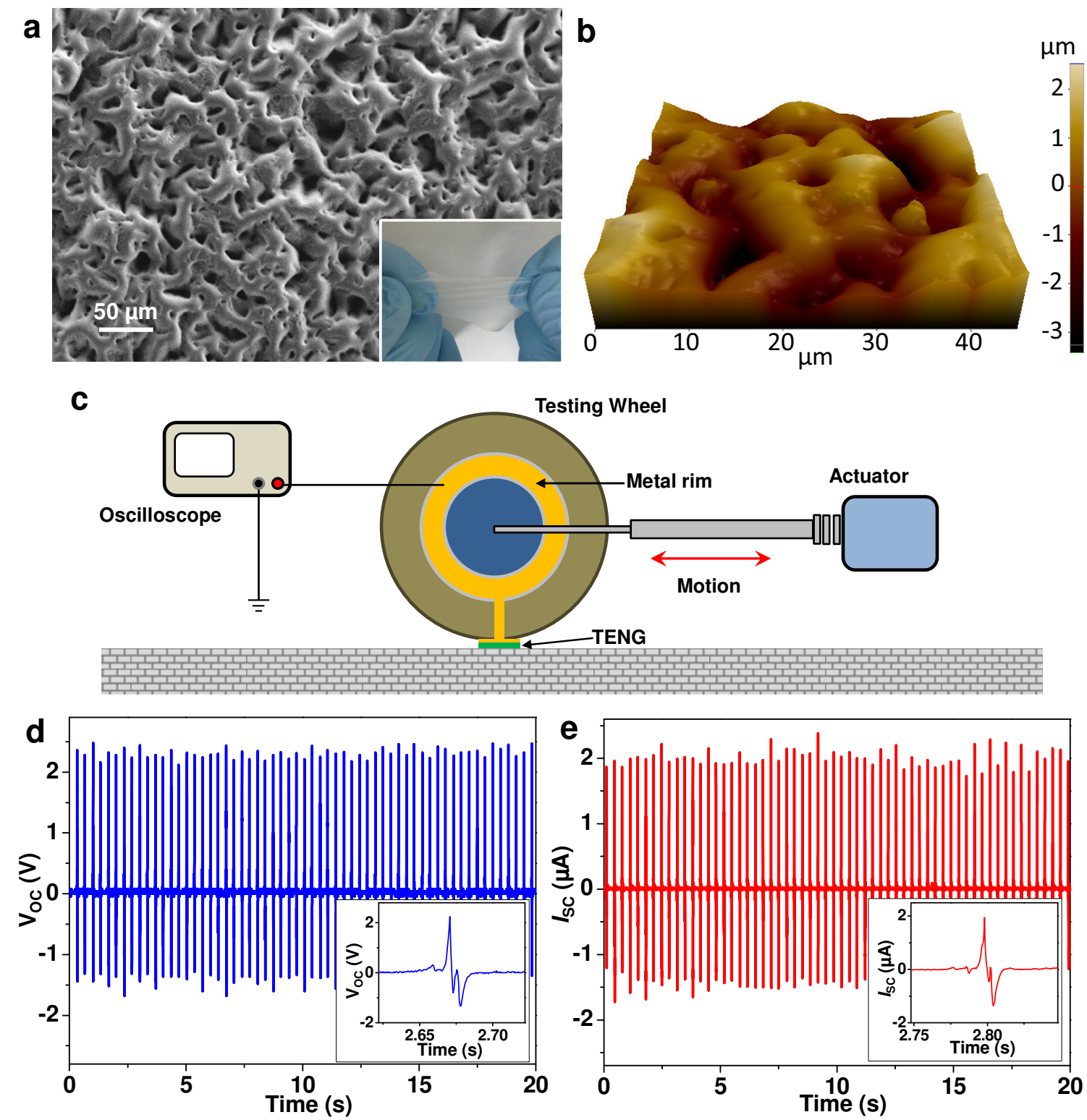

Testing Wheel

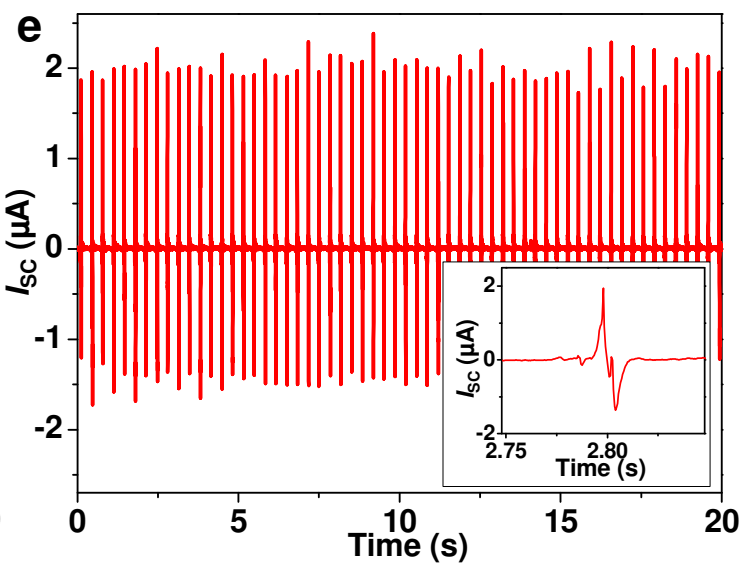

Figure 1. Design and performance of a PDMS S-TENG device on a wheel. (a) SEM image of a PDMS thin film with a rough surface imprinted by a sand paper. Inset shows the PDMS thin film is flexible and stretchable. (b) AFM topography of the PDMS surface showing the surface 
roughness is around $\sim 2-5 \mu \mathrm{m}$. (c) Schematic setup for characterizing the friction energy scavenging ability of the S-TENG from a rolling wheel. (d) The voltage and (e) current output curves of the S-TENG when the wheel was moving with a linear speed of $0.3 \mathrm{~m} / \mathrm{s}$. Insets show the enlarged output signal pattern during one contact-separation cycle.

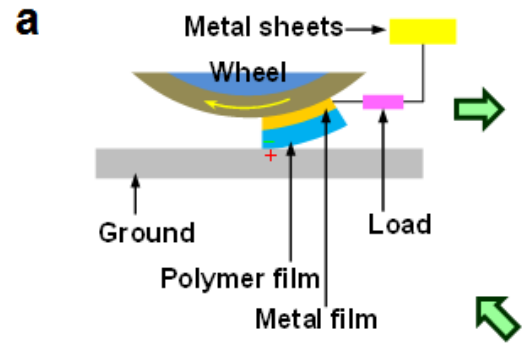

(i)

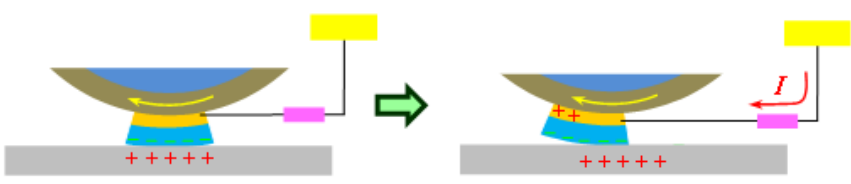

(ii)

(iii)

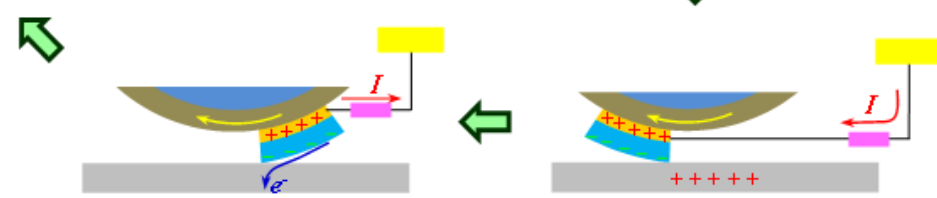

(v)
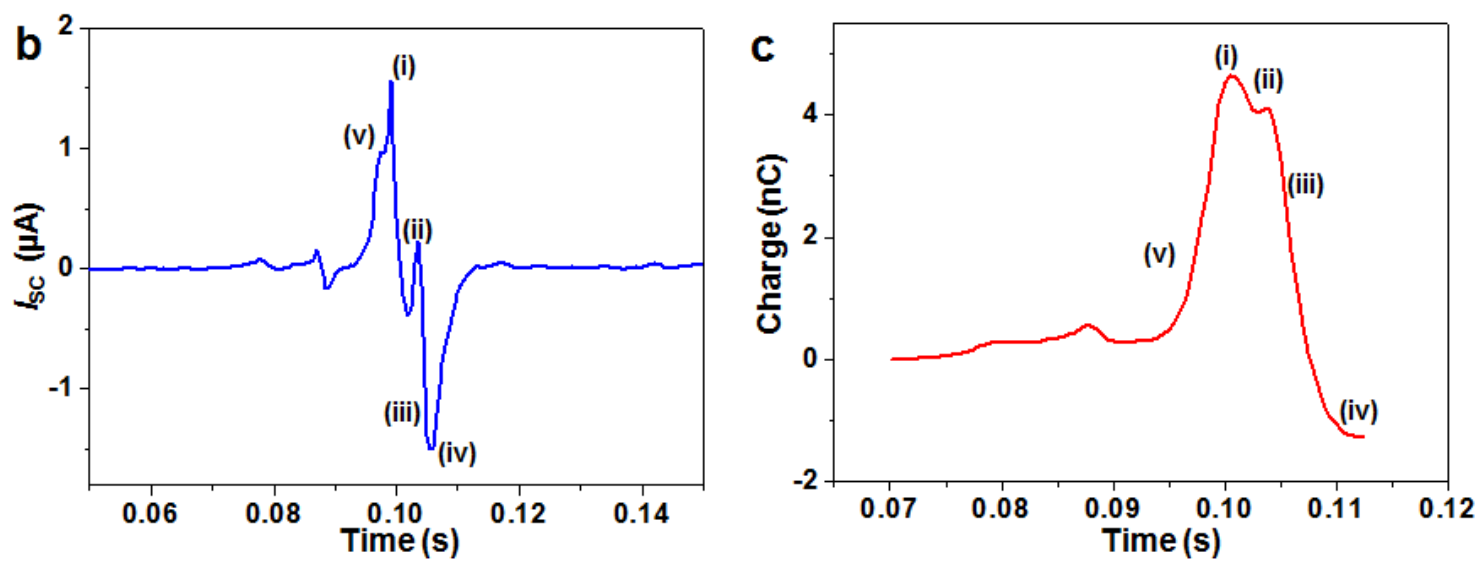

Figure 2. Working mechanism of S-TENG on wheel. (a) Schematic charge generation mechanism of the S-TENG on a rolling wheel in contact with the ground. (b) Current output signal generated from one friction cycle of the S-TENG. (c) The corresponding induced charge calculated from the integral area of the I-t curve in (b). 

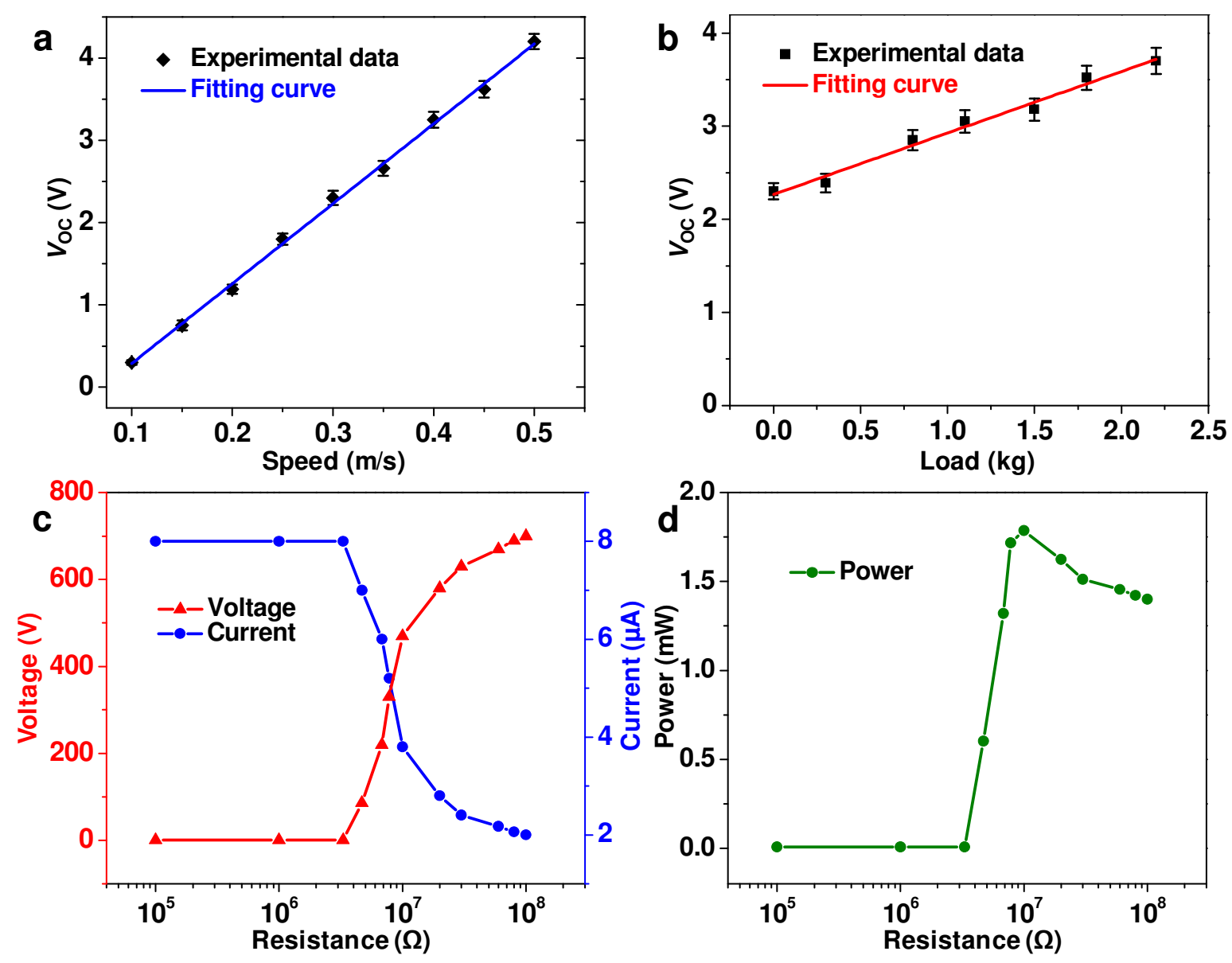

Figure 3. Investigation of the output performance of the S-TENG. (a) The relationship between the peak $V_{\mathrm{OC}}$ and average speed of the wheel. (b) The peak $V_{\mathrm{OC}}$ of the S-TENG under different extra weight loads on the wheel. The voltage was measured at the same linear speed of $0.3 \mathrm{~m} / \mathrm{s}$. (c) The output voltage and current versus the loading resistance. (d) The output power as a function of the loading resistance. 

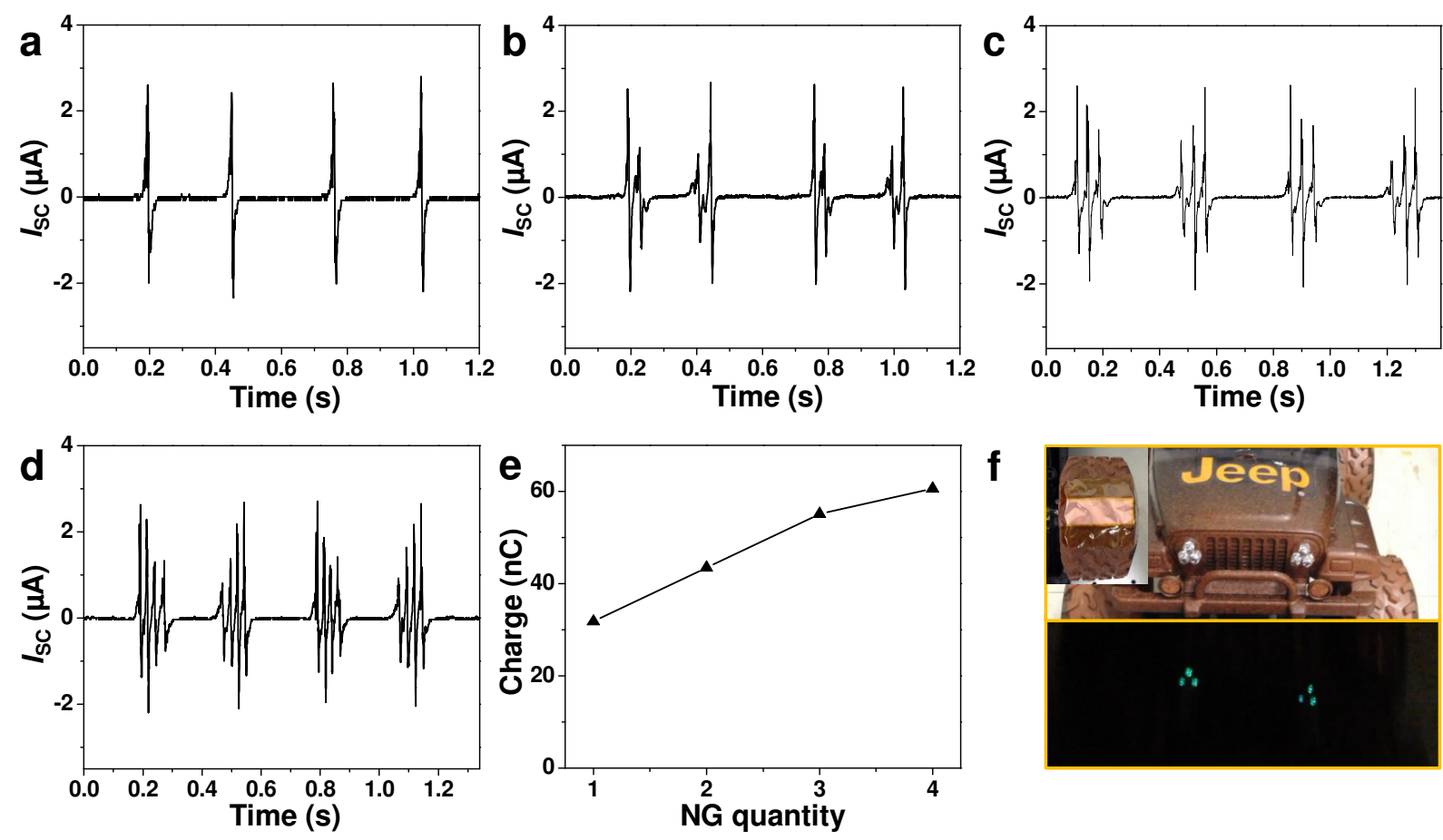

Figure 4. Electric outputs of multiple S-TENGs and the demonstration of the S-TENG on vehicle. Current outputs of the S-TENGs with (a) 1, (b) 2, (c) 3, and (d) 4 PDMS unit(s) attatched on the same wheel. (e) Plot of the total absolute charge generated in one friction cycle versus the number of PDMS unit. (f) 6 commercial green LEDs connected in series were designed as headlights of a toy vehicle. Inset shows a S-TENG $\left(1.5 \times 3.5 \mathrm{~cm}^{2}\right.$ each $)$ was attatched onto the tire surface. (g) 6 green LED headlights were lighted when the vehicle moving on the ground with a certain amount of additional force was applied to it. 


\section{References and Notes}

[1] W. Wu, L. Wang, Y. Li, F. Zhang, L. Lin, S. Niu, D. Chenet, X. Zhang, Y. Hao, T.F. Heinz, J. Hone, Z.L. Wang, Nature, 514 (2014) 470.

[2] G. Zhu, J. Chen, T. Zhang, Q. Jing, Z.L. Wang, Nat. Commun., 5 (2014) 3426.

[3] G. Zhu, Y.S. Zhou, P. Bai, X.S. Meng, Q. Jing, J. Chen, Z.L. Wang, Adv. Mater., 26 (2014) 3788.

[4] B.J. Hansen, Y. Liu, R. Yang, Z.L. Wang, ACS Nano, 4 (2010) 3647.

[5] C. Sun, J. Shi, D.J. Bayerl, X. Wang, Energy Environ. Sci., 4 (2011) 4508.

[6] X. Wang, J. Song, J. Liu, Z.L. Wang, Science, 316 (2007) 102.

[7] T.C. Hou, Y. Yang, Z.H. Lin, Y. Ding, C. Park, K.C. Pradel, L.J. Chen, Z. Lin Wang, Nano Energy, 2 (2013) 387.

[8] Q. Jing, G. Zhu, W. Wu, P. Bai, Y. Xie, R.P.S. Han, Z.L. Wang, Nano Energy, 10 (2014) 305.

[9] Y. Xie, S. Wang, S. Niu, L. Lin, Q. Jing, J. Yang, Z. Wu, Z.L. Wang, Adv. Mater., 26 (2014) 6599.

[10] S. Wang, L. Lin, Z.L. Wang, Nano Energy, 11 (2015) 436.

[11] S. Bai, L. Zhang, Q. Xu, Y. Zheng, Y. Qin, Z.L. Wang, Nano Energy, 2 (2013) 749.

[12] G. Zhu, C. Pan, W. Guo, C.Y. Chen, Y. Zhou, R. Yu, Z.L. Wang, Nano Lett., 12 (2012) 4960.

[13] Y. Yang, H. Zhang, Y. Liu, Z.H. Lin, S. Lee, Z. Lin, C.P. Wong, Z.L. Wang, ACS Nano, 7 (2013) 2808.

[14] W. Tang, Y. Han, C.B. Han, C.Z. Gao, X. Cao, Z.L. Wang, Adv. Mater., 27 (2015) 272.

[15] U.S. Department of Energy, http://www.fueleconomy.gov/feg/atv.shtml, 2015.

[16] F.R. Fan, Z.Q. Tian, Z. Lin Wang, Nano Energy, 1 (2012) 328.

[17] Z.H. Lin, G. Cheng, Y. Yang, Y.S. Zhou, S. Lee, Z.L. Wang, Adv. Funct. Mater., 24 (2014) 2810.

[18] M.B. Starr, J. Shi, X. Wang, Angew. Chem. Int. Ed. , 51 (2012) 5962.

[19] G. Cheng, Z.H. Lin, L. Lin, Z.L. Du, Z.L. Wang, ACS Nano, 7 (2013) 7383.

[20] Z.H. Lin, G. Zhu, Y.S. Zhou, Y. Yang, P. Bai, J. Chen, Z.L. Wang, Angew. Chem. Int. Ed., $52(2013) 5065$.

[21] G. Cheng, Z.H. Lin, Z. Du, Z.L. Wang, Adv. Funct. Mater., 24 (2014) 2892.

[22] Z.H. Lin, G. Cheng, L. Lin, S. Lee, Z.L. Wang, Angew. Chem. Int. Ed., 52 (2013) 12545.

[23] G. Zhu, J. Chen, Y. Liu, P. Bai, Y.S. Zhou, Q. Jing, C. Pan, Z.L. Wang, Nano Lett., 13 (2013) 2282.

[24] H. Zhang, Y. Yang, X. Zhong, Y. Su, Y. Zhou, C. Hu, Z.L. Wang, ACS Nano, 8 (2014) 680.

[25] Tires and Passenger Vehicle Fuel Economy, Transportation Research Board, Special Report 286, National Academy of Sciences, Washington, D.C., 2006. 

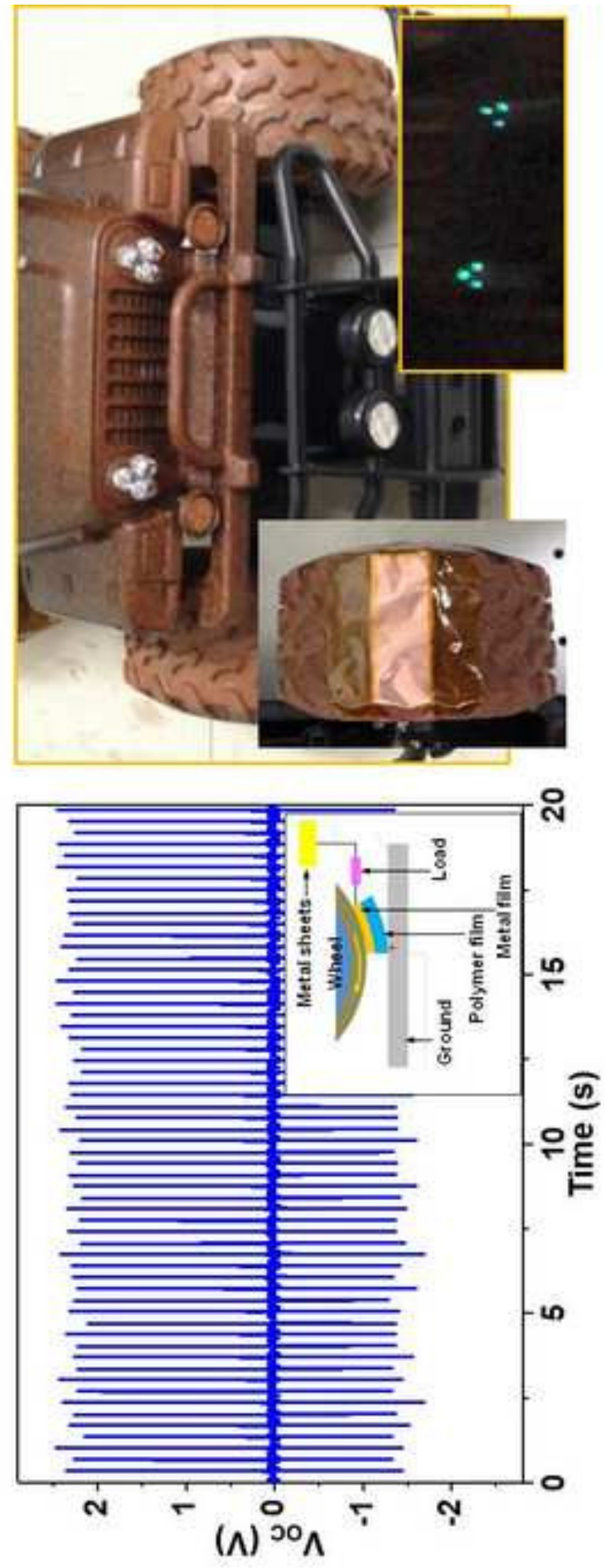